\title{
Development of the Diamond based Proton Beam Monitor for COMET Experiment
}

\author{
Yuki Fujii*广 \\ High Energy Accelerator Research Organization, KEK \\ E-mail: vfujiiepost.kek.jp
}

The COMET experiment searches for the muon-to-electron $(\mu-e)$ conversion with a sensitivity below $10^{-16}$ which is 10,000 times better than the present upper limit. This process is strictly forbidden in the standard model because of the lepton flavor conservation law. In contrast, its branching ratio can be sizable around $10^{-15}$ in many models of physics beyond the standard model (BSM). Therefore the discovery of the $\mu$-e conversion should be unambiguous evidence of BSM. Since sufficient amount of muons can be collected owing to the world most powerful pulsed proton beam at J-PARC, the background suppression is the most important to achieve the target sensitivity. In COMET, the measurement will be done between 500-1000 ns after coming the beam bunch to highly suppress the beam related prompt background. Even in this case, signals can be detected because of muon's long life time ( $\left.\tau_{\mu} \sim 800 \mathrm{~ns}\right)$ in a muonic-atom in case of using aluminum. In this scheme, the "extinction factor", (三(\#)f residual protons between two bunches)/(\# of protons in a bunch)), must be less than $10^{-9}$. To ensure such an extremely low extinction factor during the data taking, an innovative diamond detector will be adopted since it has high radiation tolerance to an intensive proton beam, and a fast time response to identify a single proton after the prompt beam. Recently, a prototype detector was developed based on a single-crystal diamond with a metal-insulator-metal type structure to perform the direct proton measurement inside the abort beam-line of J-PARC main ring. The installation was completed in this Spring and the direct beam measurement was conducted with the high intensity pulsed proton beam and the low intensity continuous beam. In this paper, results of above measurements are reported together with prospects.

EPS-HEP 2017, European Physical Society conference on High Energy Physics

5-12 July 2017

Venice, Italy

* Speaker.

${ }^{\dagger}$ On behalf of the COMET collaboration 


\section{Introduction}

In order to search for the $\mu$-e conversion with an unprecedented sensitivity below $10^{-16}$, 10,000 times better than the current upper limit[四, it is essential to realize the "extinction factor" (三(\#of residual protons between two bunches)/(\#of protons in a bunch)) less than $10^{-9}$. Such an extremely small extinction factor is already achieved at J-PARC Main-Ring(MR) by combining the RF-chopper scraping and the kicker time shifting method[[]]. However, the extinction factor should be monitored continuously during the physics data taking to ensure the extinction not to mimic the signal. The requirements of the extinction monitor are as follows: (1) high radiation tolerance to withstand an $O\left(10^{12-13}\right) \mathrm{Hz}$ proton beam, (2) fast time response to discriminate the single proton coming $500 \mathrm{~ns}$ after the main bunch, (3) low mass materials to minimize the heating and residual radiation dose around the detector, and it is not possible for ordinary detectors (e.g. scintillators or silicon detectors) to satisfy those requirements. Whereas, the diamond based detector can fulfill them due to its strong radiation tolerance and the fast time response[[B]. Recently, the prototype diamond detector consists of ultra low-mass materials to install inside the J-PARC MR beam-line was newly developed and the direct proton beam measurement was conducted.

\section{Measurements}

\section{Experimental Setup}

The diamond detector consists of the single crystal CVD(Chemical Vapor Deposited) diamond ( $4 \mathrm{~mm} \times 4 \mathrm{~mm}, 0.5 \mathrm{mmT}$ with a $2 \mathrm{~mm} \phi$ rounded metal contact), the aluminum contact plate and the graphite base plate as shown in Fig. $\mathrm{W}$. The detector is attached on the head of the linear insertion

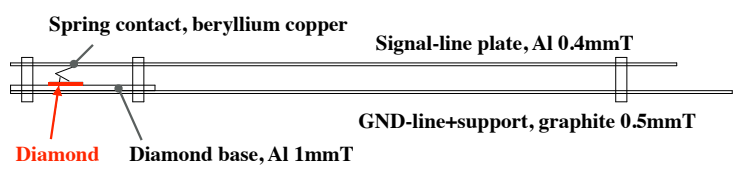

Figure 1: A schematic view of the diamond detector.
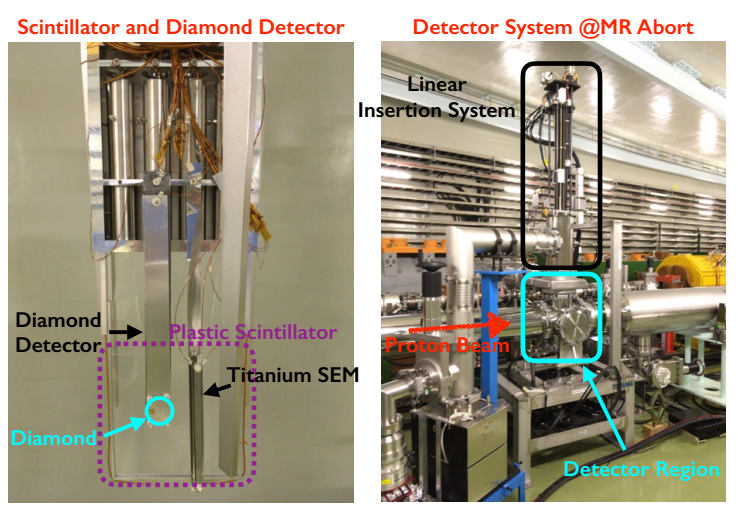

Figure 2: Photos of the diamond detector with a plastic scintillator (Left) and the detector system at the abort beam-line (Right).

system together with the plastic scintillator used in the previous extinction measurement, and the insertion system is installed inside the "abort" beam-line at J-PARC MR as shown in Fig. చ.

\section{Off-axis measurment}

To validate the detector functionality before the direct proton measurement, the indirect beam was measured by locating the detector $120 \mathrm{~mm}$ away from the beam axis. In this measurement, clear 
signals due to the secondary particles caused by the beam loss was observed as sown in Fig. [1]. The diamond detector shows shorter decay time compared to the plastic scintillator as expected.
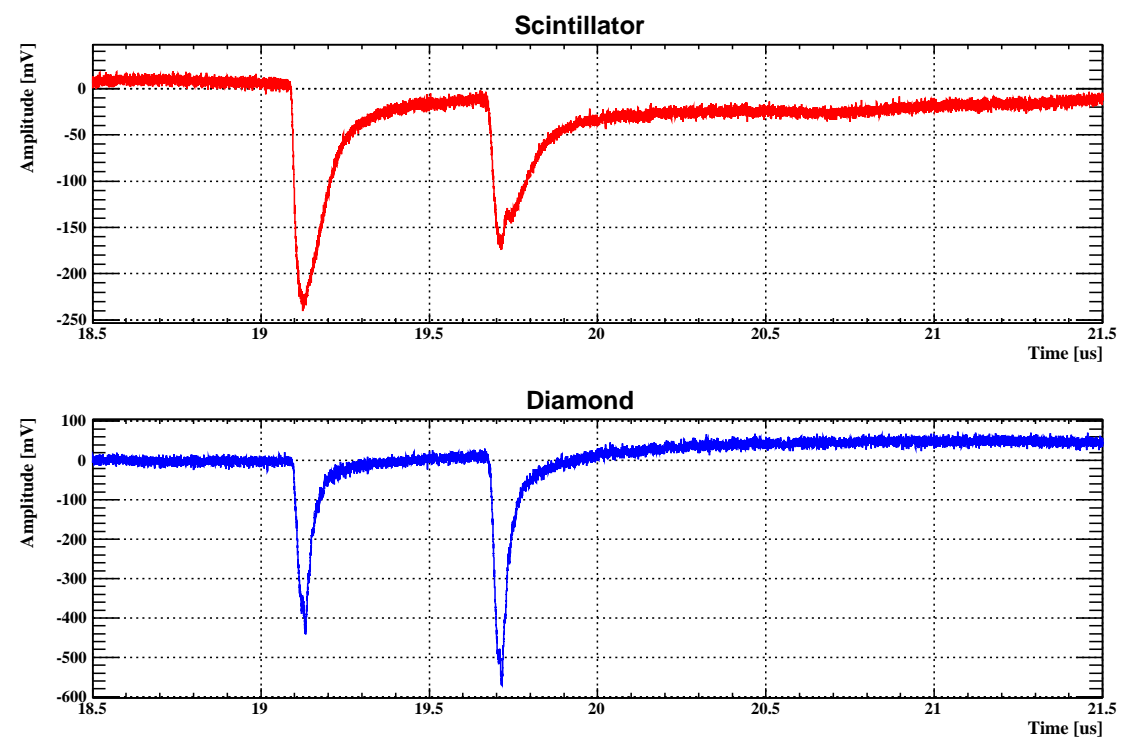

Figure 3: A comparison of the scintillator waveform (top) and the diamond waveform (bottom) using secondaries due to 2 bunches of the pulsed proton beam.

\section{Low intensity Measurement}

Since the purpose of the extinction monitor is to discriminate a few protons between bunches, the detector should be sensitive to a single proton. In order to estimate the discrimination power for the single proton, the low intensity beam measurement was performed with the slow extraction (SX) operation mode. During the SX period, more than $99.9 \%$ of protons are extracted into the J-PARC hadron experiments facility and remaining protons are withdrawn toward the abort beam dump. Thus the detection of small number of protons continuously distribute the MR one cycle $\sim 5 \mu \mathrm{s}$ is available at the abort beam-line. To observe those low intensity protons, the broadband RF amplifier, SHF $88(G=40 \mathrm{~dB})$ was used. The expected signal height is an $O(1) \mathrm{mV}$ with a few nanoseconds rise time including the cable attenuation.

\section{High Intensity Irradiation}

To estimate the performance degradation of the diamond detector caused by the high intensity proton beam, more than 200 shots of beam with $\approx 2 \times 10^{14}$ protons/shot were irradiated on the detector region during the accelerator maintenance period. The total amount of protons irradiated on the diamond is calculated to be roughly an $O\left(10^{13-14}\right)$ protons $/(4 \mathrm{~mm} \times 4 \mathrm{~mm})$. After the irradiation, the detector was removed from the abort beam-line and the detector performance will be measured.

\section{Results}

In the low intensity measurement, discrete pulses within the expected time window are observed as shown in Fig. 团. The rise time is calculated to be $\sim 5 \mathrm{~ns}$ and the minimum pulse height is a few $\mathrm{mV}$, consistent with an expected signal from the single proton. After the high intensity 

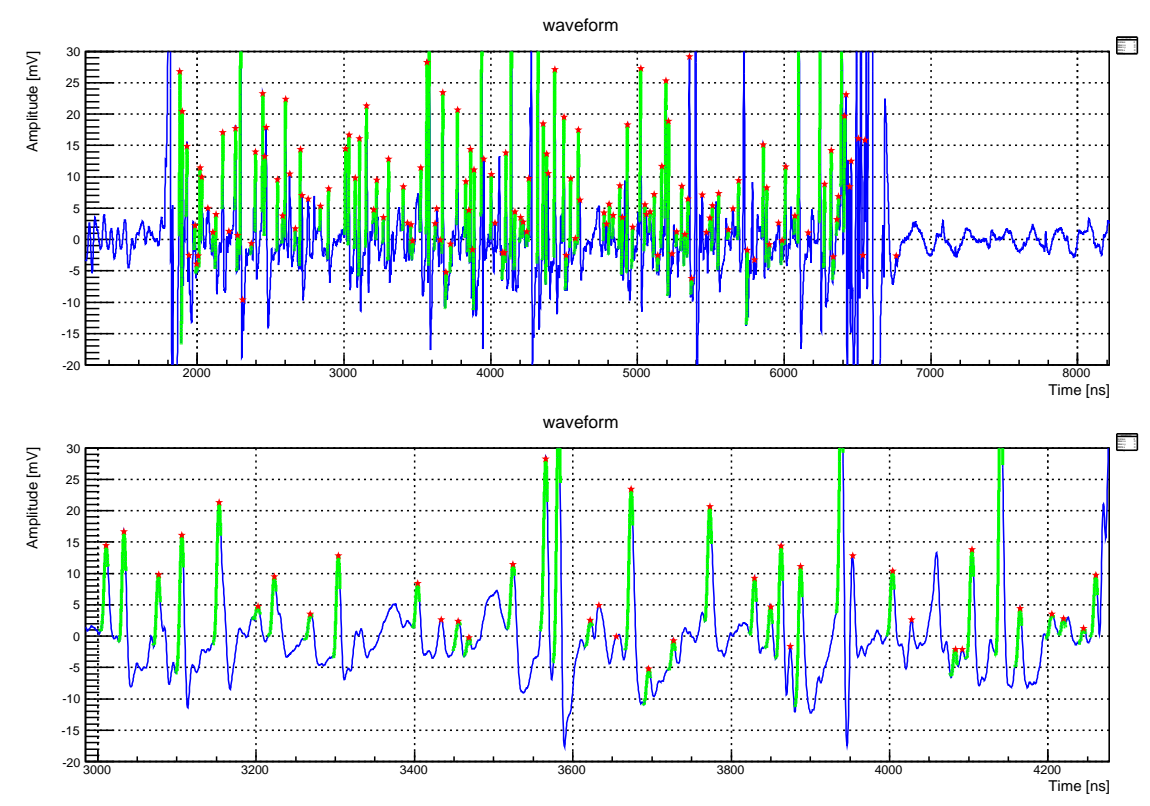

Figure 4: An example of the waveform from the diamond detector (top) and the same waveform with different time window (bottom).

irradiation, the detector was taken out from the abort-line to evaluate the performance degradation.

\section{Summary and Prospects}

The diamond based proton beam monitor is being developed for COMET to search for the $\mu-e$ conversion with a sensitivity below $10^{-16}$. The direct proton beam measurement was performed in 2017 spring/summer with the prototype detector. Discrete pulses characterized as few protons' signal are observed in the low intensity measurement and the precise analysis is ongoing to conclude the result. The high intensity beam irradiation was conducted up to an $O\left(10^{13-14}\right)$ protons/diamond and the comparison before/after the irradiation will be done soon. All measurements were done by using $30 \mathrm{GeV}$ proton beam in parallel with the normal SX and the fast extraction at the accelerator maintenance. Therefore, additional measurements with $8 \mathrm{GeV}$ bunched SX proton beam same as the COMET should be done by using an upgraded detector with a better performance.

\section{Acknowledgement}

This research is supported by a grant-in-aid, JSPS KAKENHI 16H00876 (Japan).

\section{References}

[1] W. H. Bertl et.al.[SINDRUM-II Collaboration], Eur. Phys. J. C47 (2006) pp.337-338

[2] H. Nishiguchi, "The COMET experiment: A search for muon-to-electron conversion at J-PARC", ICHEP 2014, Valencia, Spain, July 2014.

[3] W. Adam et.al.[RD42 Collaboration], Nucl. Instrum. Meth. A 476 (2002) pp.686-693 